Original Paper http://ajol.info/index.php/ijbcs http://indexmedicus.afro.who.int

\title{
La biodiversité des parcelles habitées en zone périurbaine à Kinshasa: déterminants socio-biophysiques et représentations
}

\author{
Kouagou Raoul SAMBIENI ${ }^{1,5,6,7 *}$, Apollinaire BILOSSO MOYENE ${ }^{2}$, \\ Mireille Scholastique TOYI ${ }^{3}$, Emmanuel SAMBIENI ${ }^{4}$, Armand Kuyéma NATTA ${ }^{5}$, \\ Rita OCCHIUTO ${ }^{6}$ et Jan BOGAERT ${ }^{1,7}$
}

\author{
${ }^{1}$ Ecole Régionale Post-Universitaire d'Aménagement et de Gestion Intégrés des Forêts et Territoires \\ Tropicaux (ERAIFT), Université de Kinshasa (UNIKIN), BP 15 373, Kinshasa, République Démocratique du \\ Congo. \\ ${ }^{2}$ Faculté des Sciences Agronomiques, Université de Kinshasa, BP 117 Kinshasa XI, Mont-Amba/Lemba, \\ Kinshasa, République Démocratique du Congo. \\ ${ }^{3}$ Laboratoire d'Ecologie Appliquée, Faculté des Sciences Agronomiques (FSA), Université d'Abomey-Calavi \\ (UAC), 05 BP 1752 Cotonou, Benin. \\ ${ }^{4}$ Faculté de Lettres, Arts et Sciences Humaines (FLASH), Université de Parakou, 02 BP 979 Parakou, Bénin \\ ${ }^{5}$ Laboratoire d'Ecologie, de Botanique et de Biologie Végétale (LEB), Faculté d'Agronomie, Université de \\ Parakou, 03 BP 125, Parakou, Bénin. \\ ${ }^{6}$ Laboratoire Ville-Territoire-Paysage (LabVTP), Faculté d'Architecture, Université de Liège (ULiège), 1, rue \\ Courtois-4000 Liège, Belgique. \\ ${ }^{7}$ Gembloux Agro-Bio Tech, Université de Liège, Passage des Déportés, 2 B-5030, Gembloux, Belgique. \\ "Auteur correspondant ; E-mail : skraoul@gmail.com ; Tél: (+243) $898566061 /(+229) 63957403$
}

\section{REMERCIEMENTS}

Nous remercions la Plateforme Afrique Centrale de l'Université de Liège et la Wallonie Bruxelles Internationale pour leur appui financier.

\section{RESUME}

Les zones périurbaines de Kinshasa présentent une luxuriante végétation principalement privée mais menacée par des coupes incontrôlées. Pour sa meilleure conceptualisation et gestion, la présente étude propose une analyse des déterminantes socio-biophysiques et des représentations de la végétation présente dans les parcelles habitées dans une commune périurbaine de la ville, à travers le terme proposé d' " usage du végétal pour l'habitat (UVH) ». Conduite dans la commune de Kisenso, l'étude a consisté à relever les UVH présents dans 442 parcelles ainsi que les espèces végétales les composant et les représentations associées. La richesse moyenne des parcelles en UVH obtenue est de 3 avec un coefficient de variation de 22,2\%. La zone géomorphologique de la parcelle, la province d'origine de son propriétaire et le nombre de ménages y vivant se révèlent être les principaux déterminants de la pratique de la plupart des UVH identifiés. Si les représentations associées aux UVH s'avèrent pour une grande part positive, elles intègrent cependant très peu les enjeux paysagers et environnementaux de la grande échelle. Ces résultats ouvrent un cadre de réflexion sur la meilleure prise en compte et la valorisation multifonctionnelle de la biodiversité des parcelles habitées en zone urbaine et périurbaine.

() 2018 International Formulae Group. All rights reserved.

Mots clés: biodiversité urbaine, espace périurbain, habitat, végétation domestique, perception. 


\title{
Biodiversity of inhabited plots in peri-urban zone of Kinshasa: socio- biophysics determinants and representations
}

\begin{abstract}
The peri-urban zones of Kinshasa present a luxuriant vegetation mainly private but threatened by uncontrolled cuts. For its best conceptualization and management, this study proposes an analysis of the sociobiophysics determinants and representations of the biodiversity of inhabited plots in a peri-urban municipality of Kinshasa city, through the suggested expression of "usage of vegetal for habitat (UVH) ". Performed in the municipality of Kisenso, the study consisted of identifying the findable UVH in 442 plots like the plant species composing them and the associated representations. The average UVH richness of the plots obtained is 3 with a variation coefficient of $22.2 \%$. The geomorphological area of the plot, the owner origin province and the number of households living there are the main determinants of the practice of most identified UVH. While the representations associated to UVH are largely positive, they don't include much of the landscape and environmental stakes of the broad scale. These results open a reflection framework on the best taking into account and multifunctional valorization of biodiversity of inhabited plots in the urban and peri-urban areas. (C) 2018 International Formulae Group. All rights reserved.
\end{abstract}

Keywords: urban biodiversity, suburban space, habitat, domestic vegetation, perception.

\section{INTRODUCTION}

Tout comme dans ces trois dernières décennies, l'Afrique connaîtra une forte croissance urbaine portant la proportion urbaine de sa population de $27 \%$ en 1980 à plus de 50\% en 2050 (CEA et PNUE, 2015). Cette croissance urbaine va de pair avec le développement de territoires périurbains (Bogaert et Halleux, 2015) et l'augmentation de besoins divers dont celui de l'habitat durable (Polorigni et al., 2015; UN Habitat, 2016). De ce fait, le « verdissement multifonctionnel » des villes africaines est à l'ordre de jour dans tous les agendas du développement urbain durable (FAO, 2010; Hammer et al., 2011; UNHabitat, 2016). Il est en effet, reconnu le rôle crucial de la végétation en ville pour faire face, entre autres, aux enjeux de sécurisation alimentaire et d'adaptation ou d'atténuation des changements climatiques (Kanda et al., 2014 ; Chalot, 2015 ; Koubouana et al., 2016), de gestion des inondations et érosions (Rey et al., 2004 ; Dasylva et al., 2017) et d'aménagement paysager (Cameron et al., 2012). Mieux, plusieurs études soulignent l'importance des formes d'utilisation du végétal dans les parcelles habitées comme les jardins domestiques dans la conservation de la biodiversité (Kabir et Webb, 2008). Il est donc important de reconnaître et d'analyser tous les « usages du végétal pour l'habitat (UVH) » c'est-à-dire toutes les formes d'aménagement de la parcelle habitée à base de la végétation vivante telles que les jardins, les haies vives, les plantations d'arbres domestiques, les pelouses, les toits verts, les murs végétalisés, les noues végétales, etc. Ceci est encore plus urgent sur les territoires périurbains. De fait, ils constituent les espaces qui recevront la forte urbanisation annoncée en Afrique (Butler et Spencer, 2010).

Les études sur les UVH dans le monde sont légion dans la littérature. Cependant, en Afrique, elles se concentrent presque exclusivement sur la caractérisation socioéconomique et floristique des jardins domestiques (Kabir et Webb, 2008) et leur contribution dans l'alimentation des ménages. Les haies vives, par exemple, ne sont évoquées que pour la caractérisation des systèmes agroforestiers (Yossi et al., 2004). Pourtant, l'intérêt d'étudier les différentes sortes d'UVH, les représentations associées, les facteurs déterminants de leur composition suivant les contextes socio-culturels, est démontré dans plusieurs études menées pour la plupart dans les villes des pays développés (Cook et al., 2012; Ortiz-Sanchez et al., 2015). En outre, Luginbühl (2012) insiste sur 
la nécessité de saisir les représentations sociales des choses car elles constituent le moteur de l'action. Les représentations étant entendues comme l'image mentale, les fonctions, les motivations et/ou les significations associées aux choses.

Kinshasa, la troisième mégapole d'Afrique, compte parmi les villes d'Afrique centrale au paysage périurbain dégradant du fait d'une forte urbanisation incontrôlée depuis les Indépendances (Misilu Mia et al., 2010). De plus, les communes périurbaines de Kinshasa comme celle de Kisenso sont installées sur des sites érodables et inondables (Kayembe Wa Kayembe et Wolff, 2015). Plusieurs études recommandent alors à Kinshasa divers UVH pour l'aménagement adéquat des parcelles périurbaines à base de la végétation (Wouters et Wolff, 2010). Il est, en effet, reconnu depuis déjà les années 90 que la maîtrise de l'érosion à Kinshasa devrait passer par la gestion de ce qui se passe au niveau des parcelles (Miti et al., 2004). Fort heureusement, d'éparses études reportent divers UVH dans ces communes périurbaines (Makumbelo et al., 2005). Certaines études précisent même que la quasi-totalité de la luxuriante végétation visible sur les photographies aériennes et les images satellitaires de ces communes périurbaines serait concentrée dans les parcelles habitées (SOSAK, 2014). Ce constat est d'autant plus vrai que les tissus périurbains se développent sans vides et espaces collectifs. La pérennité de cette végétation est cependant hypothéquée $\mathrm{du}$ fait non seulement de son caractère essentiellement privé mais aussi de la pratique courante de coupe pour, par exemple, alimenter les nombreuses boulangeries informelles de la ville en bois de feu (Lelo Nzuzi, 2008). Il importe donc de mieux conceptualiser cette végétation présente dans les parcelles habitées et de bien maitriser ses facteurs déterminants pour assurer sa gestion.

La présente étude propose alors une analyse des UVH observés à Kinshasa, particulièrement dans la commune périurbaine de Kisenso. Cette commune fait, en effet, face à de nombreux dégâts et risques environnementaux (érosion, inondation) du fait de sa géomorphologie à risque (collines, plaines marécageuses). De plus, elle abrite des populations en majorité pauvre faisant face à de sérieux défis de sécurité environnementale et alimentaire (ActionAid, 2012). L'étude consiste spécifiquement à (i) déterminer les déterminants socio-biophysiques qui influencent la diversité en UVH des parcelles d'une part et la pratique ou non de chacun des UVH identifiés d'autre part; (ii) évaluer la diversité des espèces composant chacun des UVH tout en mettant en évidence les plus dominantes et enfin à (iii) étudier les représentations associées à chacun des $\mathrm{UVH}$ par les populations.

\section{MATERIEL ET METHODES Milieu d'étude}

L'étude a été conduite à Kisenso, une des communes périurbaines de Kinshasa. Elle jouit d'un climat tropical humide du type AW4 selon la classification Köppen-Geiger (Peel et al., 2007) (Figures 1 et 2). Cette commune s'étend sur une superficie de 16,6 $\mathrm{km}^{2}$ subdivisée en 17 quartiers populaires. Elle compte un total d'au moins 23.846 parcelles habitées (Rapport de commune, 2015). Elle est installée sur un site collinaire dont le sol est à dominance sablo-limoneux et donc d'une extrême érodabilité accentuée par l'urbanisation incontrôlée et la destruction du couvert végétal originel (Kayembe Wa Kayembe et Wolff, 2015). La végétation jadis constituée de savanes steppiques, n'est actuellement constituée que de la végétation des parcelles habitées (Miti et al., 2004; Rapport de commune, 2015). La population estimée à 359.510 habitants, est constituée à plus de $80 \%$ d'enfants et de jeunes gens de 0 à 42 ans (Rapport de commune, 2015). De plus, la grande majorité de la population exerce un métier manuel et/ou le petit commerce.

\section{Collecte de données}

La collecte des données s'est faite durant le mois d'avril 2017 correspondant à un des mois de pic pluviométrique dans la zone et donc à la période de pleine végétation (Figure 1). Elle s'est déroulée en deux phases. La première a consisté en une enquête 
exploratoire et test du guide d'entretien conduite dans 30 parcelles choisies de façon aléatoire. L'unité enquêtée est double, la parcelle et un de ses habitants. Nous définissons la parcelle comme une portion de terrain munie d'un propriétaire. Les habitants enquêtés sont ceux ayant des droits sur les usages du végétal pour l'habitat (UVH) identifiés dans la parcelle correspondante incluant les droits de décision sur leur gestion et leur composition et pas nécessairement des droits fonciers. En seconde phase, nous avons utilisé les données d'enquête exploratoire pour déterminer la proportion $(\mathrm{p}=90 \%)$ de parcelles présentant au moins un UVH et conséquemment la taille d'échantillon ( $n$ ) suivant l'approximation normale de la distribution binomiale (Dagnelie, 1998):

$$
n=\frac{\mathbf{u}_{1-\alpha / 2}^{2} \times \mathbf{p}(\mathbf{1}-\mathbf{p})}{\mathbf{d}^{2}} \text {, où } U_{1-\alpha / 2}=1,96 \text { est la }
$$

valeur de la variable aléatoire normale pour un risque $\alpha$ égale à $5 \% ; d$ est la marge d'erreur d'estimation de paramètres issus de l'enquête fixée à $5 \%$.

La valeur de $n$ obtenue est d'environ 139. Nous nous sommes ainsi théoriquement résolus d'enquêter 150 parcelles dans chacune des trois zones géomorphologiques considérées (Tableau 1). Suivant les réalités de terrain, un total de 442 parcelles a été effectivement parcouru dont 145 en zone de plateau, 149 en plaine et 148 en flanc.

Les données collectées sur la base d'une fiche d'enquête ont porté sur les caractéristiques socio-biophysiques des parcelles (Tableau 1); les différents UVH présents dans chaque parcelle et les noms des espèces les composant. En plus, nous avons recueilli les représentations associées à chacun des UVH relevés dans chaque parcelle auprès d'un de ses habitants en même temps que ses données socio-économiques (Tableau 2).

\section{Analyse statistique}

La diversité des UVH a été analysée à travers la richesse des parcelles en UVH qui représente le nombre d'UVH employés sur la parcelle. Afin de déterminer l'effet des caractéristiques socio-biophysiques des parcelles sur leur richesse en UVH, nous avons procédé à une analyse de variance suivant le Modèle Linéaire Généralisé ou Generalized Linear Model (GLM) (Forthofer, 2007). Pour mieux mettre en évidence les résultats de cette analyse de variance, nous avons représenté graphiquement la répartition des richesses en UVH et celle de leurs proportions d'absence/présence dans les parcelles.

Pour déterminer l'effet des caractéristiques socio-biophysiques des parcelles sur la pratique ou non de chacun des UVH, nous avons réalisé une analyse de régression logistique binaire. Les variables indépendantes étaient les caractéristiques socio-biophysiques des parcelles (Tableau 1) et la variable dépendante, la présence ou non de l'UVH correspondant dans la parcelle. Pour ce faire, nous avons considéré toutes les interactions possibles dans les modèles initiaux. Ils ont été, par la suite, simplifiés par la procédure pas à pas descendante. Nous avons sélectionné le meilleur modèle grâce au Critère d'Information d'Akaike (AIC) (Akaike, 1974). Les analyses ont été effectuées sous le logiciel R.

Par ailleurs, nous avons principalement utilisé la richesse spécifique (totale, minimale, maximale et moyenne et le coefficient de variation associé) pour évaluer la diversité des espèces composant chacun des UVH. En plus, nous avons déterminé la répartition en fréquence relative des espèces composant chaque UVH pour mettre en évidence les espèces les plus dominantes. Les noms des espèces et les informations complémentaires ont été vérifiés dans les différents documents disponibles (Pauwels, 2003 ; Latham et Konda $\mathrm{Ku}, 2007)$. Les espèces herborisées ont été identifiées à l'Herbarium de l'Université de Kinshasa.

Enfin, l'analyse des représentations associées aux UVH par les populations a consisté en premier lieu, à regrouper les propos des enquêtés dans des catégories de représentation. Pour chacune de ces dernières, nous avons en second lieu procédé au calcul des fréquences de citation correspondantes, lesquelles sont reportées graphiquement (Sambiéni et al., 2015). 


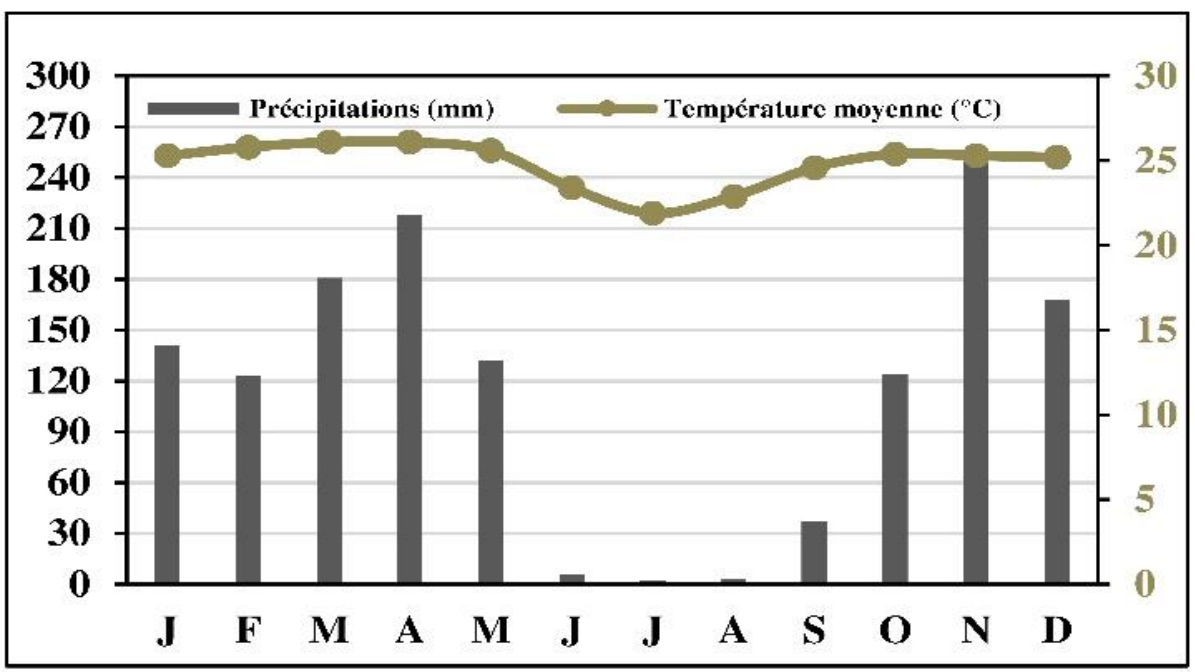

Figure 1: Diagramme climatique et courbe des températures moyennes de la commune de Kisenso. Source de données : https://fr.climate-data.org/location/48388/

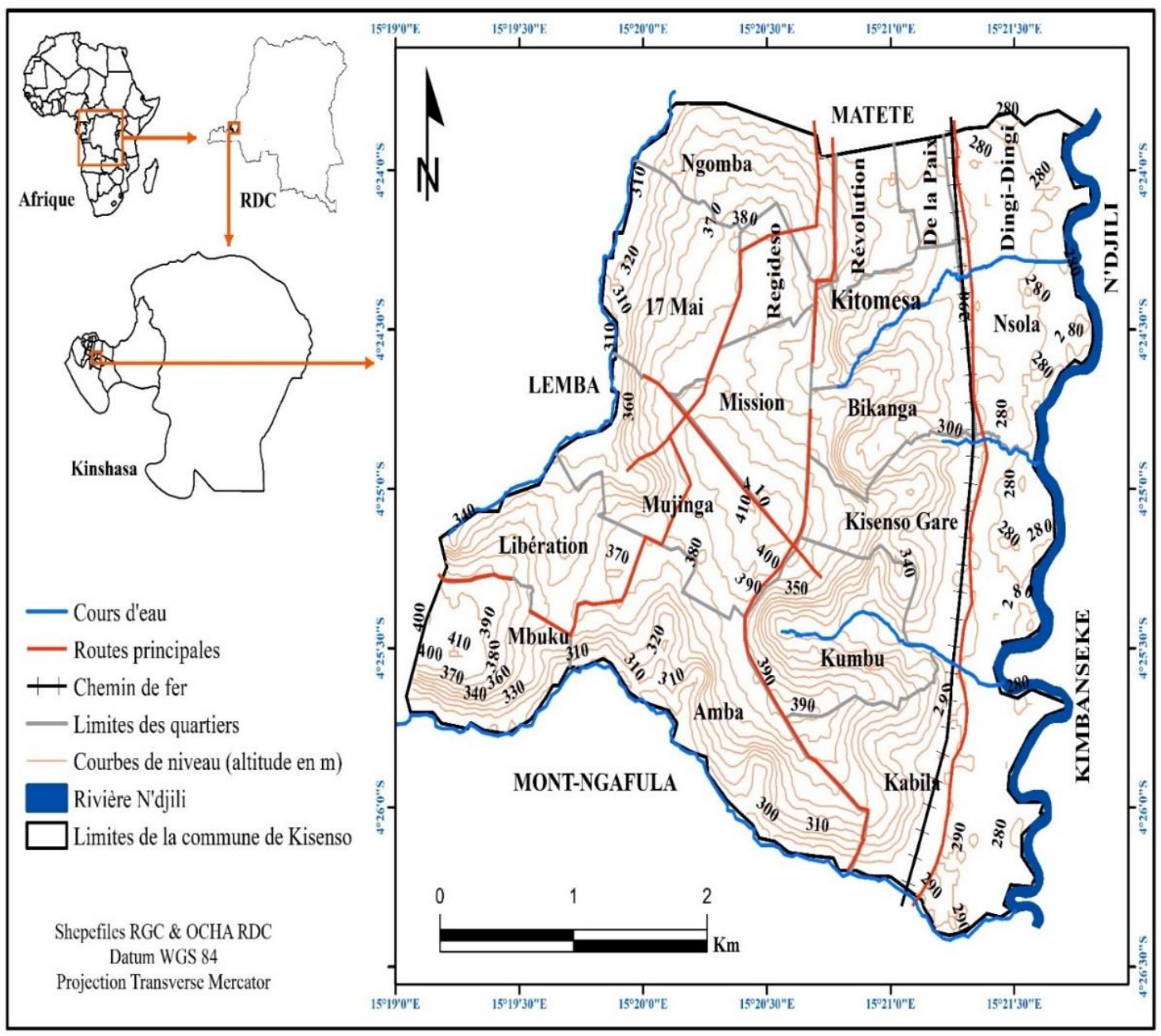

Figure 2: Carte de localisation et de subdivisions administratives de la commune étudiée. 
Tableau 1: Caractéristiques socio-biophysiques des parcelles considérées dans l'étude.

\begin{tabular}{lcl}
\hline Intitulés & Abréviations & Modalités \\
\hline Province d'origine du propriétaire & POP & Modaliltés non fixées \\
\hline Statut des occupants & SO & Propriétaire uniquement; locataire uniquement; mixte \\
\hline Nombre de ménages & Nmge & Faible (1 à 2); moyen (3 à 4); Elevé (> 5) \\
\hline Zone géomorphologique & ZoneGeo & Plateau, flanc, plaine \\
\hline Etendue de la parcelle $\left(\mathrm{m}^{2}\right)$ & Eparcelle & $\begin{array}{l}\text { E1 (100 à 150); E2 (151 à 300); E3 (301 à 500); E4 } \\
(>500)\end{array}$ \\
\hline Coefficient d'occupation de la parcelle & COP & COP1 (0 à 20); COP2 (21 à 40); COP3 (41 à 60); \\
(proportion de la surface bâtie en \%) & & COP4 (61 à 80); COP5 (81 à 100) \\
\hline
\end{tabular}

Tableau 2 : Présentation des caractéristiques socio-économiques des habitants enquêtés.

\begin{tabular}{|c|c|c|c|c|c|}
\hline Paramètres & Effectif & Proportion (\%) & Paramètres & Effectif & Proportion (\%) \\
\hline Genre & & & Province d'origine & & \\
\hline Masculin & 184 & 41,6 & Kongo & 188 & 42,5 \\
\hline Féminin & 258 & 58,4 & Kwilu & 105 & 23,8 \\
\hline \multirow[t]{2}{*}{ Total } & 442 & 100,0 & Kwango & 34 & 7,7 \\
\hline & & & Equateur & 22 & 5,0 \\
\hline Âge (en années) & & & Kasaï Occidental & 18 & 4,1 \\
\hline $18-25$ & 31 & 7,0 & Katanga & 14 & 3,2 \\
\hline $26-35$ & 108 & 24,4 & Maniema & 10 & 2,3 \\
\hline $36-45$ & 122 & 27,6 & Kasaï Oriental & 9 & 2,0 \\
\hline $46-55$ & 81 & 18,3 & Sud-Kivu & 8 & 1,8 \\
\hline$>56$ & 100 & 22,6 & Province orientale & 7 & 1,6 \\
\hline \multirow[t]{2}{*}{ Total } & 442 & 100,0 & Maï Ndombe & 7 & 1,6 \\
\hline & & & Sud-Ubangi & 5 & 1,1 \\
\hline \multicolumn{6}{|l|}{ Niveau } \\
\hline d'instruction & & & Ituri & 4 & 0,9 \\
\hline Sans instruction & 13 & 2,9 & Mongala & 3 & 0,7 \\
\hline Alphabétisé & 39 & 8,8 & Tshopo & 2 & 0,5 \\
\hline Primaire & 23 & 5,2 & Sankuru & 2 & 0,5 \\
\hline Secondaire & 210 & 47,5 & Nord Kivu & 2 & 0,5 \\
\hline Universitaire & 157 & 35,5 & Nord-Ubangi & 1 & 0,2 \\
\hline Total & 442 & 100,0 & Haut Lomami & 1 & 0,2 \\
\hline & & & Total & 442 & 100,0 \\
\hline
\end{tabular}


RESULTATS

\section{La diversité des usages du végétal pour l'habitat}

Dans la zone étudiée, nous distinguons un total de cinq différents usages du végétal pour l'habitat (UVH). Il s'agit du jardin ornemental, du jardin potager, de la haie vive, de la pelouse et de l'arbre domestique (Figure 3). Les résultats d'analyse de variance appliquée sur les caractéristiques sociobiophysiques des parcelles et le nombre d'UVH présents dans chaque parcelle indiquent qu'aucun des facteurs considérés n'a d'effet significatif (prob. > 5\%) sur la richesse des parcelles en UVH. Pour preuve, plus de $85 \%$ des parcelles étudiées présentent une richesse en UVH égale ou supérieure à la richesse moyenne observée soit $3 \mathrm{UVH}$ avec un très faible coefficient de variation de $22,15 \%$ (Figure 4). Nous constatons, par ailleurs, que 3 des $5 \mathrm{UVH}$ sont plus fréquentes. Il s'agit de l'arbre domestique présent dans $99 \%$ des parcelles enquêtées; du jardin potager avec $89 \%$ de fréquence d'occurrence et la haie vive avec $76 \%$ de présence (Figure 5).

\section{Les déterminants socio-biophysiques de la pratique des usages du végétal pour l'habitat (UVH)}

Les régressions logistiques appliquées aux caractéristiques socio-biophysiques des parcelles sur les données de présence-absence de chacun des UVH ont permis d'élaborer des modèles pour 4 des 5 UVH identifiés (Tableau 3). Le cinquième autre UVH, notamment l'arbre domestique, n'a présenté aucun modèle avec des effets significatifs.

Dans les modèles élaborés (Tableau 3), nous observons des effets individuels et/ou combinés de cinq principales caractéristiques socio-biophysiques des parcelles à savoir : la zone géomorphologique (ZoneGeo), la province d'origine du propriétaire (POP), le coefficient d'occupation de la parcelle (COP), le nombre de ménages (Nmge) et partiellement l'étendue de la parcelle (Eparcelle). Bien que les modèles présentent des effets significatifs jusqu'à une erreur de $10 \%$, nous ne nous focalisons que sur ceux significatifs à au moins 5\%.
Dans cette optique, la ZoneGeo est la seule variable qui a un effet significatif sur tous les quatre UVH. Nous observons qu'allant du plateau à la plaine, la pratique du jardin ornemental et de la pelouse est plus fréquente (Figure 6A et $\mathrm{D}$ ) tandis que celle du jardin potager et de la haie vive est moins fréquente (Figure 6B et $\mathrm{C}$ ).

La POP est la seconde variable qui a le plus d'effets sur les UVH, notamment sur la pelouse et la haie vive. Pour exemple, la probabilité est plus forte de trouver une pelouse dans la parcelle dont le propriétaire est originaire de la province du Maniema que de celle du Kwilu (Figure 7A et C). Par contre, pour ces mêmes provinces d'origine, l'effet est contraire pour la haie vive (Figure 7B).

Par ailleurs, nous notons que plus le Nmge est élevé plus la probabilité d'usage de la haie vive est faible (Figure 8A). L'interaction des variables Nmge et COP d'une part et celle de POP et COP d'autre part présentent également des effets significatifs sur la pratique de la haie vive (Figure 9B et C).

\section{La diversité des espèces utilisées pour les différents usages du végétal pour l'habitat (UVH)}

Les richesses spécifiques moyennes de chacun des UVH sont faibles. Elles varient entre 1 et environ 5 espèces. La pelouse est l'UVH qui indique la plus faible richesse spécifique moyenne ( $\mathrm{N}_{\text {moy }}=1$ espèce) alors que le jardin potager est celui qui en affiche la plus forte $\left(\mathrm{N}_{\text {moy }} \approx 5\right.$ espèces) (Tableau 4 ). Pour chaque UVH, les faibles valeurs du coefficient de variation (entre $36 \%$ et $46 \%$ ) indiquent qu'au niveau des parcelles, les richesses spécifiques afférentes sont pour une grande part proche de la moyenne (Tableau 4).

Par ailleurs, nous observons que la pratique des UVH mobilise un nombre non négligeable d'espèces végétales, soit au total 125 espèces réparties dans 105 genres et 55 familles (Tableau 4). La plantation d'arbres domestiques est celle qui mobilise le plus grand nombre d'espèces avec une richesse spécifique totale de 43 espèces. Elle est suivie par le jardin potager avec une richesse 
spécifique totale de 31 espèces. La pelouse affiche raisonnablement la plus faible richesse spécifique totale (3 espèces). Elle est, en effet, en pratique monospécifique. En outre, pour chaque UVH, nous notons que seule une à au plus trois espèces dominent par leurs plus fortes fréquences (Tableau 5).

\section{Les représentations associées aux usages du végétal pour l'habitat (UVH)}

Les représentations associées à chacun des UVH sont très variables, et pour une grande part, positives. Pour chaque UVH, il y a une représentation qui est unanimement évoquée par presque tous les enquêtés (67\% à 100\%) (Figure 10). Il s'agit souvent d'un des premiers rôles associables à l'UVH correspondant : élément d'embellissement pour le jardin ornemental ; source de légumes pour le jardin potager ; moyen de délimitation de la parcelle pour la haie vive, moyen de lutte antiérosive pour la pelouse et enfin source de fruits comestibles pour l'arbre domestique. Toutefois, nous notons des représentations préjudiciables à la pratique à long terme de certains UVH, notamment le jardin ornemental, le jardin potager et la haie vive. En effet, pour le jardin ornemental, environ $18 \%$ d'enquêtés l'associe au niveau social des évolués laissant comprendre que pour eux, c'est une pratique réservée aux riches (Figure 10). Par contre, la pratique du jardin potager est liée à la pauvreté par $22 \%$ des enquêtés et à un moyen d'occuper le temps ou l'espace, respectivement par $31 \%$ et $27 \%$ des enquêtés (Figure 10). Concernant la haie vive, $60 \%$ d'enquêtés la perçoive comme une clôture provisoire utilisée par manque de moyens pour construire une clôture en dure.

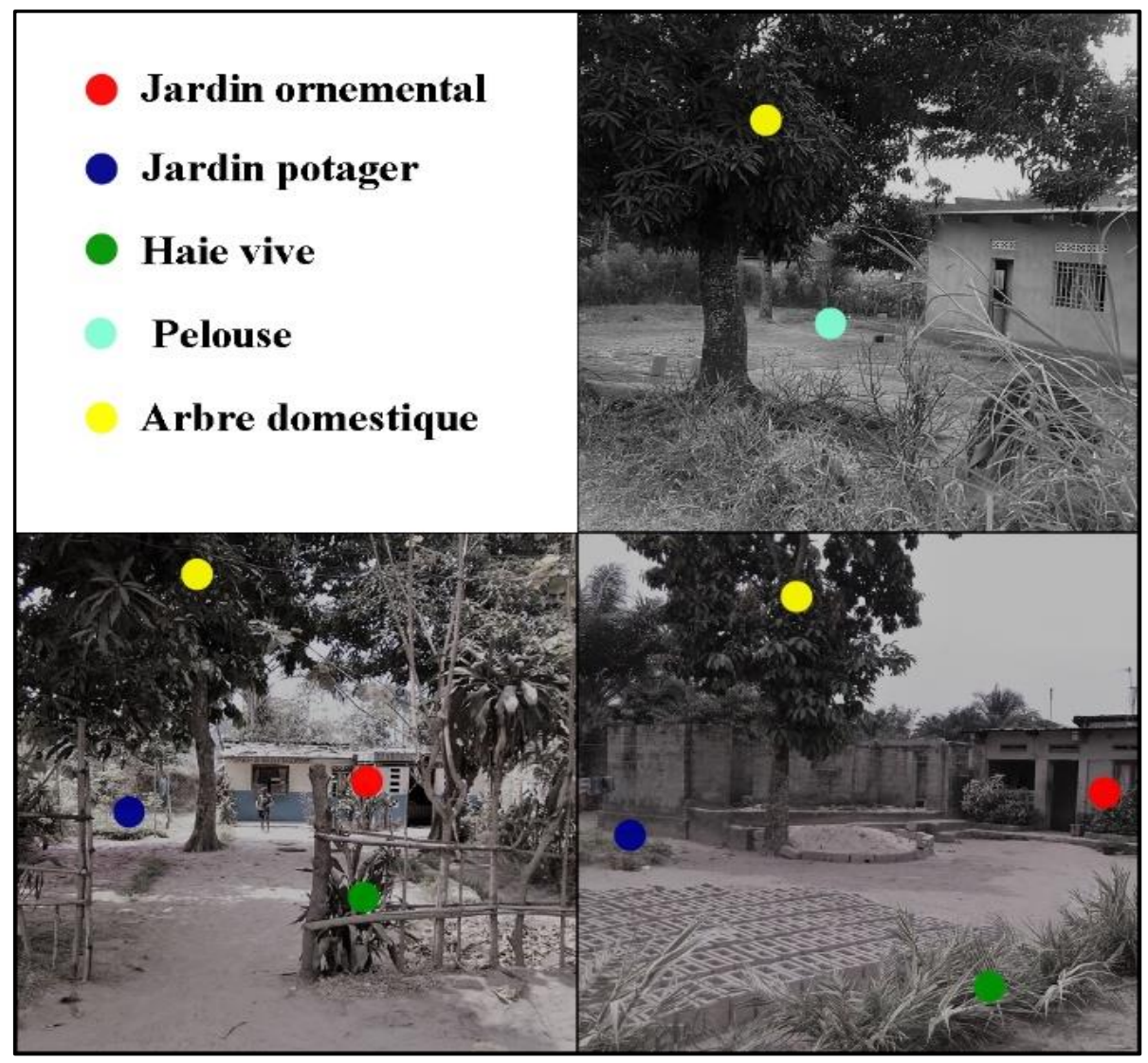

Figure 3: Illustration des différents usages du végétal pour l'habitat (UVH) observés dans la zone périurbaine étudiée. Photos: K. R. Sambiéni. 


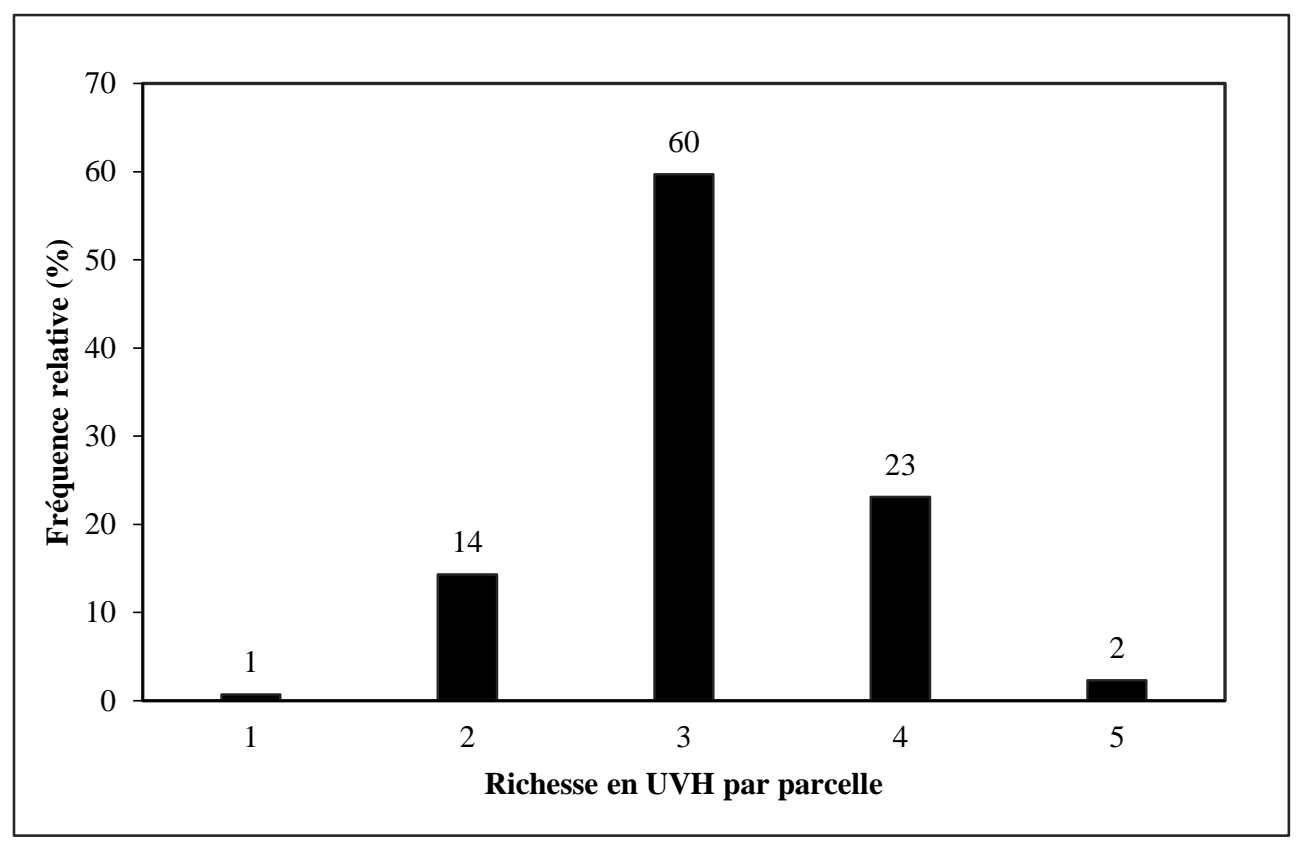

Figure 4: Répartition des richesses en usages du végétal pour l'habitat (UVH) par parcelle sur le total des 442 parcelles enquêtées. La richesse moyenne est de $3 \mathrm{UVH}$ avec un coefficient de variation de 22,15\%.

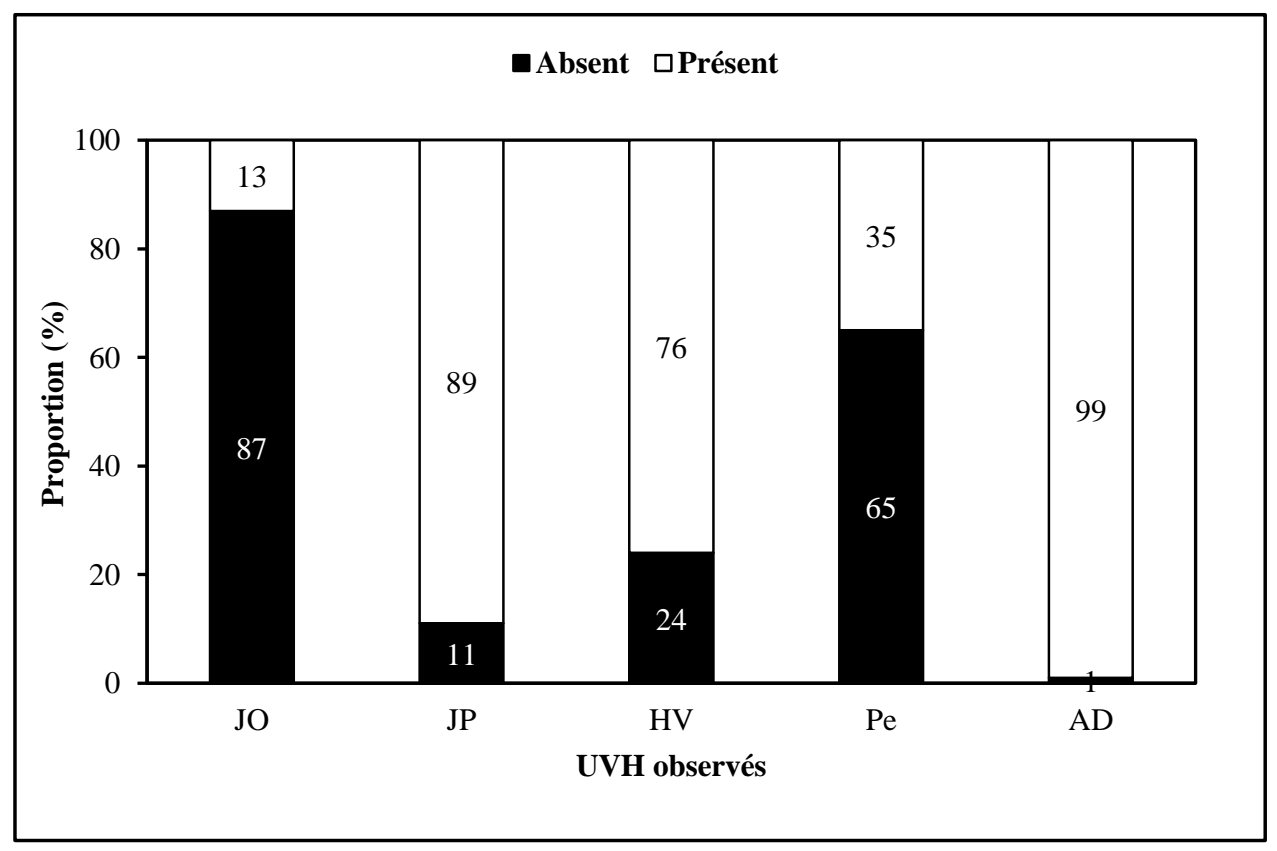

Figure 5: Répartition des proportions d'absence et de présence de chacun des usages du végétal pour l'habitat (UVH) dans les 442 parcelles enquêtées. JO : jardin ornemental ; JP : jardin potager ; HV : haie vive ; $\mathrm{Pe}$ : pelouse ; $\mathrm{AD}$ : arbre domestique. 
Tableau 3: Résultats des régressions logistiques binaires relatives à chacun des usages du végétal pour l'habitat.

\begin{tabular}{|c|c|c|c|c|}
\hline \multicolumn{5}{|c|}{ Modèle-Jardin ornemental: AIC = 299,39 } \\
\hline & Coefficient & Erreur type & Valeur de Z & Probablité(>|z|) \\
\hline Constante & $-5,0538$ & 0,6313 & $-8,006$ & $1,19 \mathrm{e}-15 * * *$ \\
\hline ZoneGeo & 1,3562 & 0,2375 & 5,711 & $1,13 \mathrm{e}-08 * * *$ \\
\hline \multicolumn{5}{|c|}{ Modèle-Jardin potager: $\mathrm{AIC}=\mathbf{2 9 4}$} \\
\hline Constante & 5,69125 & 1,92058 & 2,963 & $0,00304 * *$ \\
\hline ZoneGeo & $-0,80113$ & 0,2678 & $-2,991$ & $0,00278 * *$ \\
\hline POP & 0,12386 & 0,07389 & 1,676 & 0,09368 \\
\hline COP & $-1,03985$ & 0,57206 & $-1,818$ & 0,06911 . \\
\hline Eparcelle & $-0,74165$ & 0,65706 & $-1,129$ & 0,259 \\
\hline Eparcelle:COP & 0,36353 & 0,19135 & 1,9 & 0,05746 \\
\hline \multicolumn{5}{|c|}{ Modèle-Haie vive: $\mathrm{AIC}=\mathbf{4 7 6 , 1 9}$} \\
\hline Constante & 6,02741 & 1,36792 & 4,406 & $1,05 \mathrm{e}-05 * * *$ \\
\hline ZoneGeo & $-0,33911$ & 0,14963 & $-2,266$ & $0,02343 *$ \\
\hline POP & $-0,41416$ & 0,1363 & $-3,039$ & $0,00238 * *$ \\
\hline $\mathrm{COP}$ & $-0,9435$ & 0,39172 & $-2,409$ & 0,05601 \\
\hline Nmge & $-2,47694$ & 0,97141 & $-2,55$ & $0,01078 *$ \\
\hline POP:COP & 0,08285 & 0,04052 & 2,045 & $0,04090 *$ \\
\hline Nmge:COP & 0,58484 & 0,28284 & 2,068 & $0,03866 *$ \\
\hline \multicolumn{5}{|c|}{ Modèle-Pelouse: AIC $=567,29$} \\
\hline Constante & $-0,94886$ & 0,39427 & $-2,407$ & $0,0161 *$ \\
\hline ZoneGeo & 0,31797 & 0,15678 & 2,028 & $0,0425 *$ \\
\hline POP & 0,09301 & 0,03976 & 2,339 & $0,0193 *$ \\
\hline Eparcelle & $-0,21724$ & 0,15745 & $-1,38$ & 0,1677 \\
\hline
\end{tabular}

. = significatif à $10 \% ;^{*}=$ significatif à $5 \%$; ** = significatif à $1 \% ; * * *=$ significatif à $0,1 \%$.

Tableau 4: Richesse spécifique des différents usages du végétal pour l'habitat (UVH) identifiés.

\begin{tabular}{lccccccc}
\hline Types d'UVH & $\mathbf{N}_{\text {min }}$ & $\mathbf{N}_{\text {moy }} \pm \mathbf{E S}$ & $\mathbf{C V}(\boldsymbol{\%})$ & $\mathbf{N}_{\text {max }}$ & $\mathbf{N}_{\text {Total }}$ & $\mathbf{N}_{\text {genre }}$ & $\mathbf{N}_{\text {famille }}$ \\
\hline Jardin ornamental & 1 & $2,7 \pm 0,3$ & 46,4 & 5 & 30 & 30 & 19 \\
Jardin potager & 1 & $4,7 \pm 0,1$ & 41,4 & 13 & 31 & 31 & 18 \\
Haie vive & 1 & $1,8 \pm 0,0$ & 36,4 & 3 & 18 & 18 & 12 \\
Pelouse & 1 & $1,0 \pm 0,0$ & - & 1 & 3 & 3 & 1 \\
Arbre domestique & 1 & $2,7 \pm 0,1$ & 43,3 & 7 & 43 & 35 & 25 \\
Ensemble des UVH & $\mathbf{1}$ & $\mathbf{8 , 5} \mathbf{0 , 2}$ & $\mathbf{4 1 , 4}$ & $\mathbf{2 3}$ & $\mathbf{1 2 5}$ & $\mathbf{1 0 5}$ & $\mathbf{5 5}$ \\
\hline
\end{tabular}

$\mathrm{N}_{\min }=$ richesse minimale $; \mathrm{N}_{\text {moy }}=$ richesse moyenne $; \mathrm{ES}=$ erreur standard ; $\mathrm{CV}$ = coefficient de variation, $\mathrm{N}_{\max }=$ richesse maximale et $\mathrm{N}_{\text {Total }}=$ nombre total d'espèces inventoriées. 


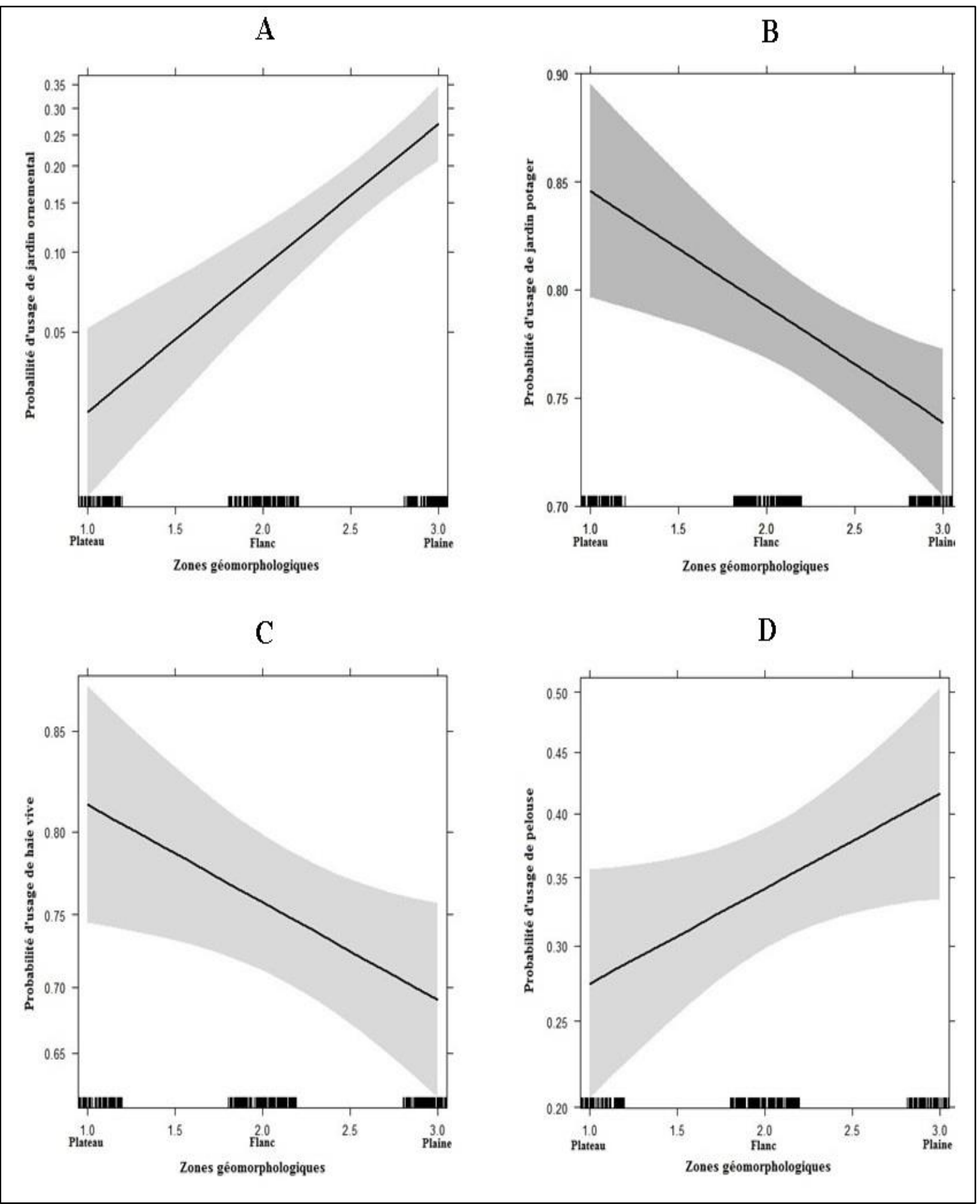

Figure 6 : Effet de la zone géomorphologique sur différents usages du végétal pour l'habitat. 


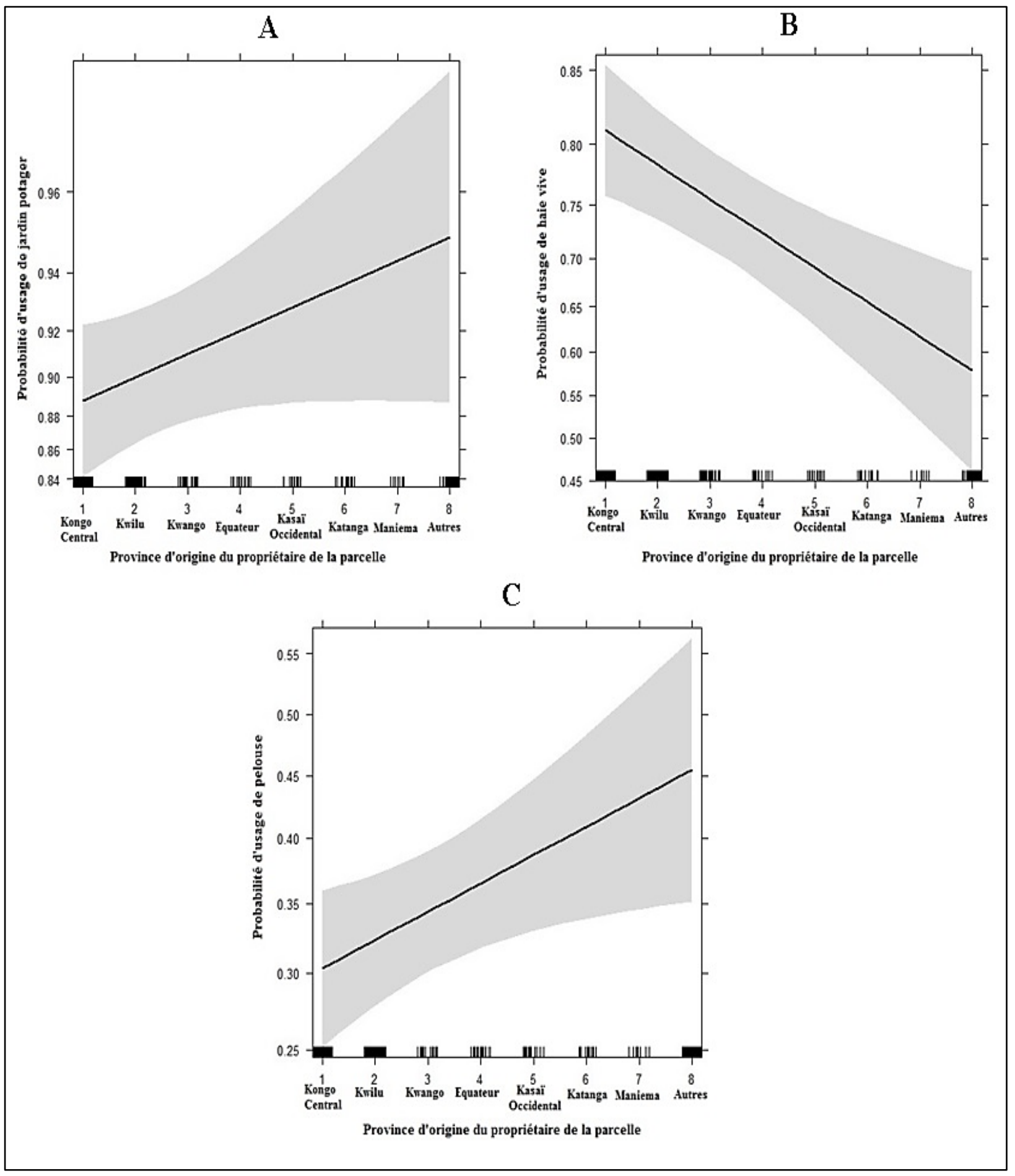

Figure 7: Effet de la province d'origine du propriétaire de la parcelle sur différents usages du végétal pour l'habitat. 


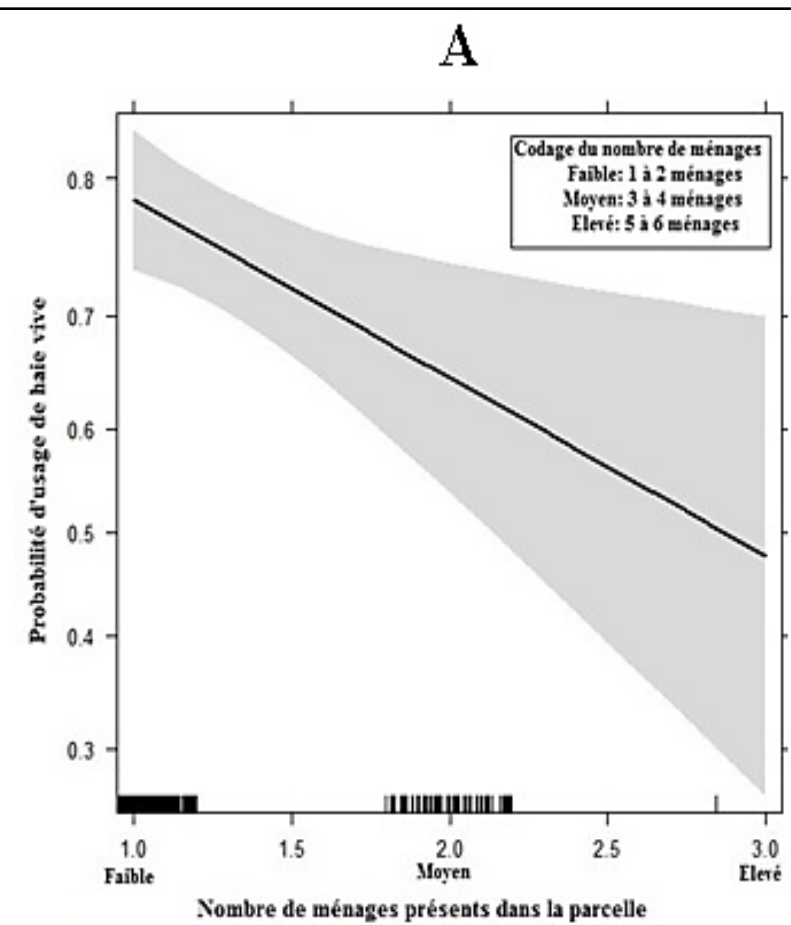

$\mathrm{C}$

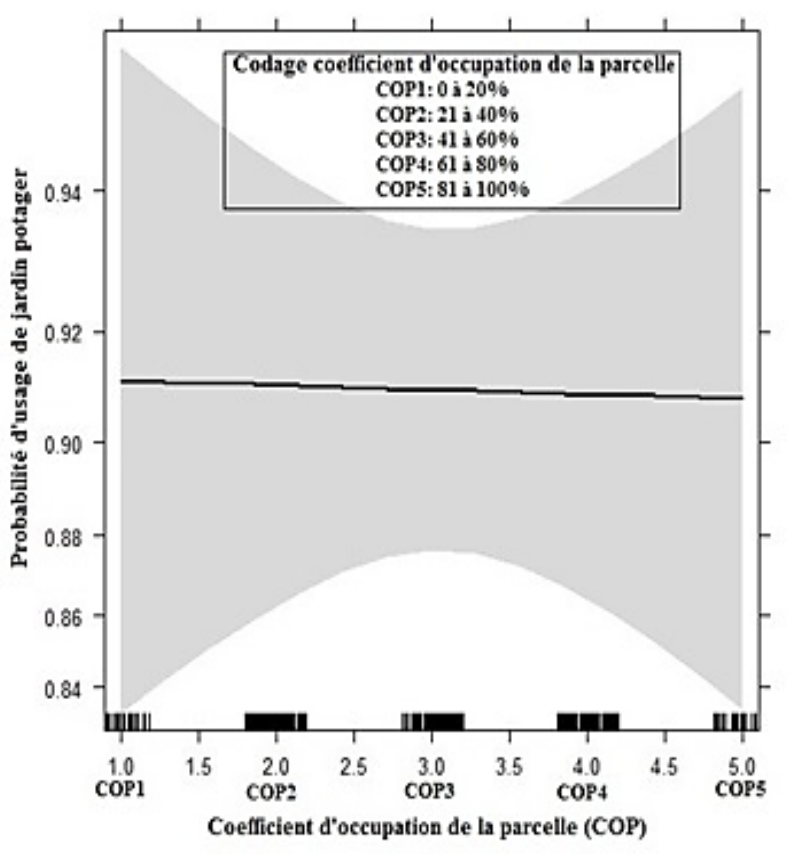

B

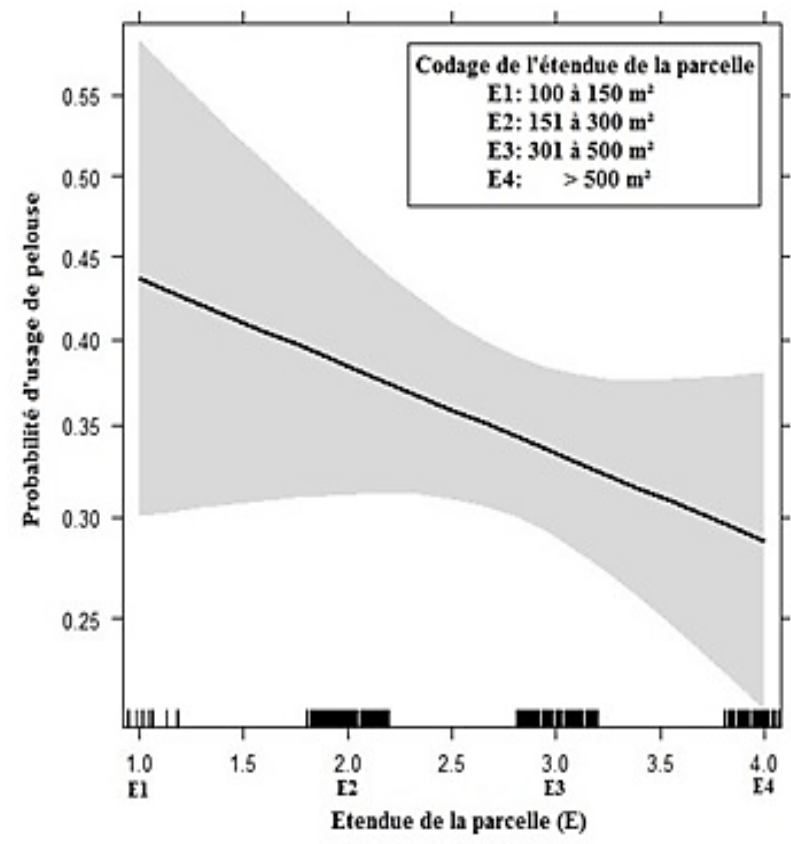

D

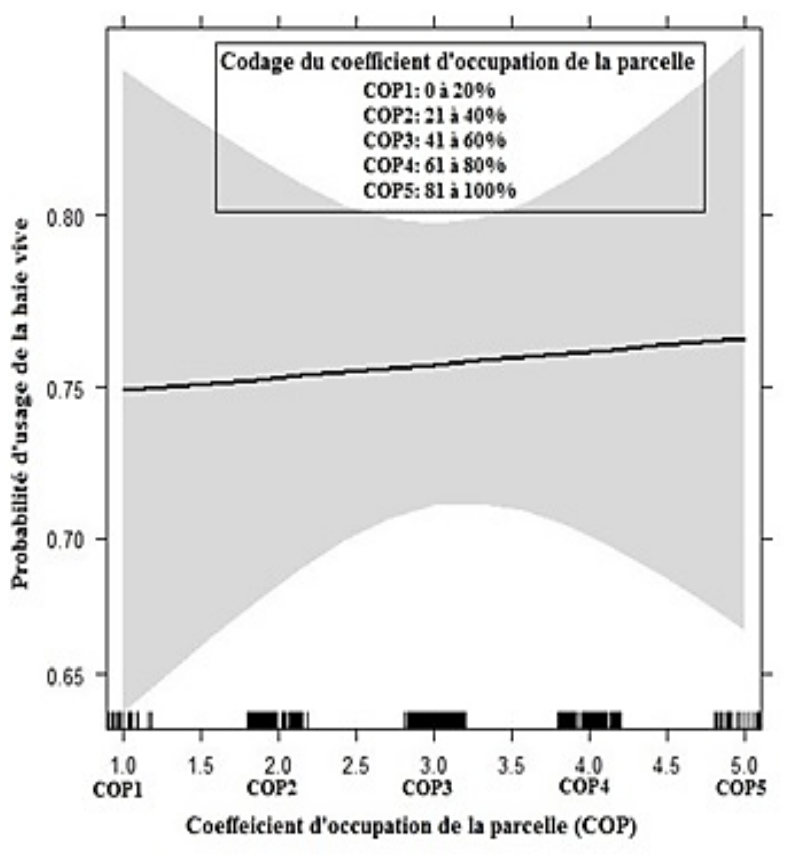

Figure 8: Effet du nombre de ménages, de l'étendue et du coefficient d'occupation de la parcelle sur différents usages du végétal pour l'habitat. 


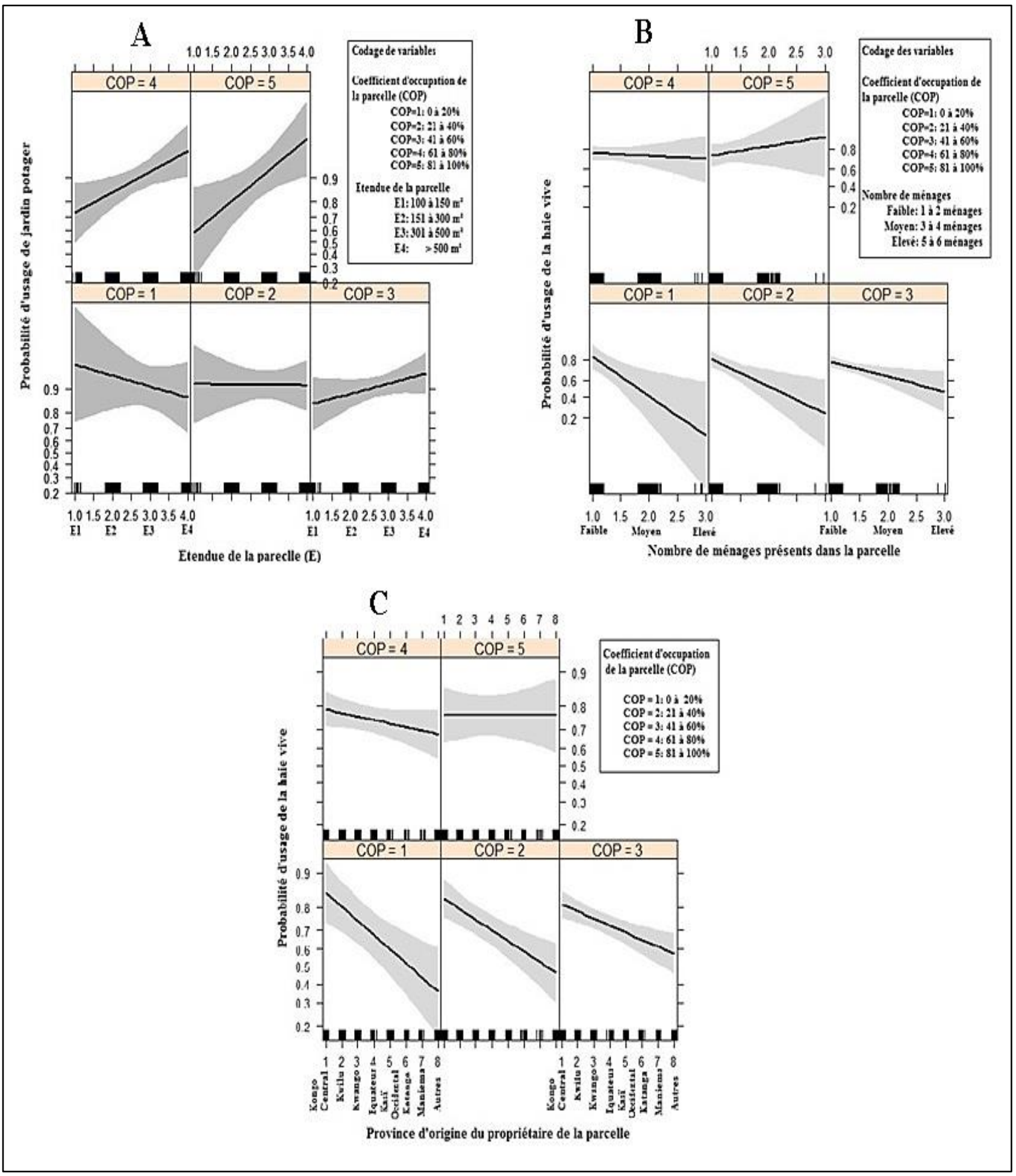

Figure 9: Effets combinés de quelques caractéristiques socio-biophysiques des parcelles sur différents usages du végétal pour l'habitat. 
Tableau 5: Présentation des espèces composant les différents usages du végétal pour l'habitat.

\begin{tabular}{|c|c|c|c|c|}
\hline Nom d'espèces & Noms en français & $\begin{array}{c}\text { Noms courant en } \\
\text { vernaculaire }\end{array}$ & Familles & $\begin{array}{c}\text { Fréquences } \\
(\%)\end{array}$ \\
\hline \multicolumn{5}{|c|}{$\begin{array}{l}\text { Espèces de jardin ornemntal } \\
\end{array}$} \\
\hline & Hibiscus Rose de & & & \\
\hline $\begin{array}{l}\text { Hibiscus rosa-sinensis L. } \\
\text { Biophytum Zenkeri }\end{array}$ & Chine & & Malvaceae & 31,8 \\
\hline Guillaumin & & & Oxalidaceae & 10,6 \\
\hline $\begin{array}{l}\text { Helianthus annuus } \mathrm{L} \text {. } \\
\text { Aglaonema pictum (Roxb.) }\end{array}$ & Tournesol & & Asteraceae & 6,1 \\
\hline Kunth & & & Araceae & 4,5 \\
\hline $\begin{array}{l}\text { Chlorophytum comosum } \\
\text { (Thunb.) Jacques }\end{array}$ & & & Asparagaceae & 4,5 \\
\hline $\begin{array}{l}\text { Chamaerops humilis } \mathrm{L} . \\
\text { Codieum variegatum (L.) }\end{array}$ & Palmier nain & & Arecaceae & 3,0 \\
\hline $\begin{array}{l}\text { A.Juss. } \\
\text { Ravenala madagascarensis }\end{array}$ & Croton & & Euphorbiaceae & 3,0 \\
\hline Sonn. & Arbre du voyageur & & Strelitziaceae & 3,0 \\
\hline 22 autres espèces & & & & 33,3 \\
\hline Total & & & & 100,0 \\
\hline \multicolumn{5}{|c|}{ Espèces de jardin potager } \\
\hline Manihot esculenta Crantz & Manioc doux & Pondu & $\begin{array}{l}\text { Euphorbiaceae } \\
\text { Convolvulacea }\end{array}$ & 24,3 \\
\hline $\begin{array}{l}\text { Ipomoea batatas (L.) Lam. } \\
\text { Hibiscus acetosella Welw. }\end{array}$ & Patate douce & Matembele & $\mathrm{e}$ & 23,2 \\
\hline Ex Hiern & Oseille & Ngai ngai & Malvaceae & 6,7 \\
\hline $\begin{array}{l}\text { Solanum esculentum Dunal } \\
\text { Colocasia esculenta (L.) }\end{array}$ & Aubergine & Solo & Solanaceae & 5,7 \\
\hline Schott & Taro & Mbala ya langa & Araceae & 5,5 \\
\hline 26 autres espèces & & & & 34,6 \\
\hline Total & & & & 100,0 \\
\hline \multicolumn{5}{|c|}{$\begin{array}{l}\text { Espèces de haie vive } \\
\end{array}$} \\
\hline $\begin{array}{l}\text { Euphorbia tirucalli } \mathrm{L} . \\
\text { Draceana fragrans }(\mathrm{L} .) \mathrm{Ker}\end{array}$ & Euphorbe Crayon & Kol & Euphorbiaceae & 44,2 \\
\hline Gawl. & & & Asparagaceae & 35,0 \\
\hline Vernonia amygdalina Delile & Vernonie commune & Mululu & Asteraceae & 5,1 \\
\hline $\begin{array}{l}\text { Bambusa vulgaris Schrad. } \\
\text { Tithonia diversifolia }\end{array}$ & Bambou & Bambo & Poaceae & 3,3 \\
\hline (Hemsl.) A.Gray & & Mikadi kadi & Asteraceae & 3,3 \\
\hline 13 autres espèces & & & & 9,0 \\
\hline Total & & & & 100,0 \\
\hline \multicolumn{5}{|c|}{ Espèces de la pelouse } \\
\hline Paspalum $s p$ & & & Poaceae & 69,9 \\
\hline $\begin{array}{l}\text { Eremochloa ophiuroides } \\
\text { (Munro) Hack.. } \\
\text { Chrysopogon zizanioides }\end{array}$ & & & Poaceae & 21,5 \\
\hline $\begin{array}{l}\text { (L.) Roberty } \\
\text { Total }\end{array}$ & Vétiver & Vetive & Poaceae & $\begin{array}{r}8,6 \\
\mathbf{1 0 0 , 0}\end{array}$ \\
\hline
\end{tabular}




\begin{tabular}{|c|c|c|c|c|}
\hline \multicolumn{5}{|c|}{ Espèces d'arbre domestique } \\
\hline Mangifera indica $\mathrm{L}$. & Manguier & Manga & Anacardiaceae & 24,3 \\
\hline Persea americana Mill. & Avocatier & Avoka & Lauraceae & 18.3 \\
\hline Carica papaya $\mathrm{L}$ & Papapyer & payi payi & Caricaceae & 10.3 \\
\hline Dacryodes edulis (G. Dc & & & & \\
\hline H.J. Lam & Safoutier & Safu & Burseraceae & 7.5 \\
\hline Elaeis guinensis Jacq. & Palmier à huile & Mbila & Arecaceae & 5.5 \\
\hline Musa parasidiaca $\mathrm{L}$. & Bananier & Bitabe & Musaceae & 5.5 \\
\hline 37 autres espèces & & & & 28.5 \\
\hline Total & & & & 100.0 \\
\hline
\end{tabular}

Seules les espèces portant les cinq premières plus fortes fréquences sont présentées.

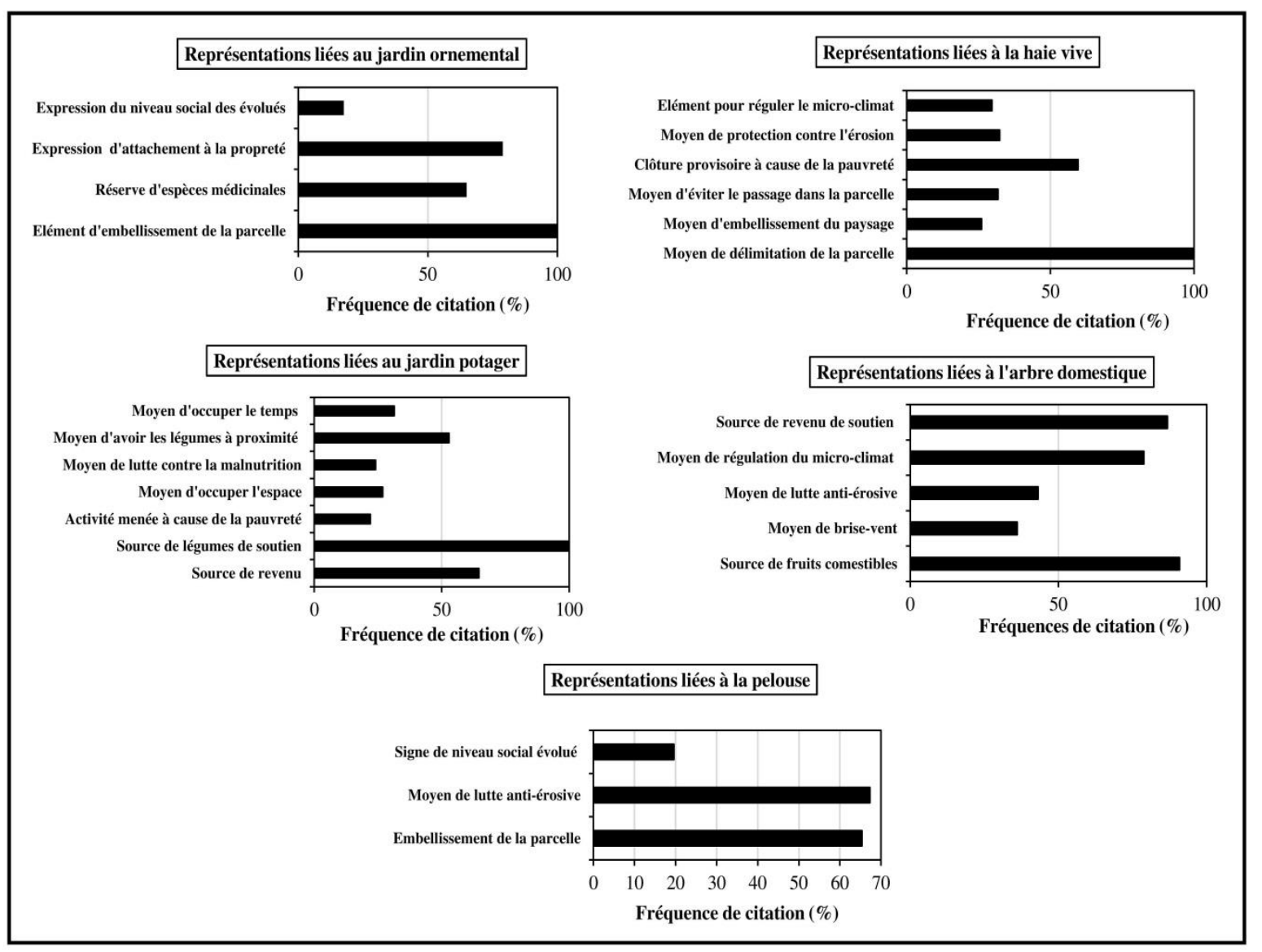

Figure 10: Répartition des représentations associées aux différents usages du végétal pour l'habitat identifiés.

\section{DISCUSSION}

Dans cette étude, cinq différents usages du végétal pour l'habitat (UVH) ont été au total identifiés avec une moyenne de trois par parcelle. La richesse de la parcelle en UVH s'est avérée déterminer par aucune des caractéristiques socio-biophysiques des parcelles considérées. Il se révèle ainsi que la pratique des UVH est généralisée dans la zone périurbaine étudiée. Ces résultats confirment le constat fait de la présence d'une luxuriante végétation en parcelles habitées dans les zones 
périurbaines de la ville de Kinshasa (Makumbelo et al., 2005; Wouters et Wolff, 2010 ; SOSAK, 2014). La grande importance accordée au végétal dans l'aménagement des parcelles dans la zone étudiée provient, entre autres, des recommandations d'aménagement végétal issues d'études reportant la forte érodabilité des sols sableux et peu cohérents des communes collinaires de Kinshasa comme celle de Kisenso, la commune étudiée (Miti et al., 2004). Ces recommandations alors érigées en règles étaient rigoureusement respectées à l'époque coloniale et un peu après les Indépendances (Lelo Nzuzi, 2008). Aujourd'hui et surtout dans les zones périurbaines installées spontanément comme la zone étudiée, la pratique des UVH est plus héritée et ou répliquée qu'imposée dans un contexte où l'Etat est quasiment démissionnaire. Nous notons, par ailleurs, que le jardin ornemental reste remarquablement rare dans la zone d'étude (Figure 5). Cette rareté est comme compensée par la forte présence des jardins potagers confirmant ainsi l'intérêt plus poussé des populations kinoises aux espèces comestibles et commerciales du fait, entre autres, de la prégnante pauvreté (Lelo Nzuzi, 2008).

La zone géomorphologique de la parcelle (ZoneGeo), la province d'origine du propriétaire (POP), le nombre de ménages (Nmge) vivant sur la parcelle et son coefficient d'occupation (COP) sont les principales caractéristiques sociobiophysiques qui influent sur la pratique de l'un ou l'autre des UVH identifiés sauf l'arbre domestique. Ce dernier UVH se révèle ainsi être celui dont la pratique est la moins influencée par les caractéristiques de la parcelle et conséquemment la plus fréquente (99\% de présence) (Figure 5). Nous observons que la haie vive et la pelouse sont plus déterminées par les caractéristiques sociales des parcelles (POP, Nmge) que celles biophysiques (ZonGéo). Toutefois, la pratique du jardin potager et ornemental se révèle plus déterminée par les caractéristiques biophysiques, notamment la ZoneGeo. Cette dernière caractéristique est celle qui se révèle influencer le plus la pratique des UVH. Ce résultat se justifie par le fait que la ZoneGeo résume bien les conditions du milieu et surtout le niveau d'exposition aux risques d'érosion ou inondation qui, pour une part non négligeable, motive les populations à pratiquer les UVH. Cependant, nous observons paradoxalement que la pratique du jardin potager décroît du plateau à la plaine. L'explication en est que les populations vivant en plaine dans la zone d'étude pratiquent plus le maraîchage à but commercial et alimentaire sur de grandes surfaces à l'extérieur des parcelles (Lelo Nzuzi, 2008).

Concernant la diversité des espèces exploitées dans la zone périurbaine, son appréciation reste relative. En effet, la commune périurbaine étudiée avec un total de 125 espèces s'avère bien plus riche que les communes urbaines de Kinshasa comme celle de Limete où Makumbelo et al. (2005) avaient identifié, dans les parcelles, un total de 35 espèces de légumes et d'arbres fruitiers. De même, en considérant l'ensemble des UVH, la richesse moyenne totale obtenue ( $\approx 9$ espèces) est bien relativement proche à celle signalée dans les zones périurbaines d'autres villes africaines comme celles du Bénin (9 à 11 espèces) (Salako et al., 2014). Par contre, des richesses moyennes bien plus élevées sont signalées sous les tropiques comme dans la ville du Nicaragua en Amérique latine avec 70 espèces en moyenne (Méndez et al., 2001). En considérant les UVH individuellement et plus particulièrement le jardin potager souvent constitué d'un à trois blocs d'environ $1 \mathrm{~m}^{2}$ en moyenne (Figure 3), la richesse moyenne obtenue (environ 5 espèces) est bien non négligeable vue la superficie occupée. Ces résultats montrent bien qu'il y a un véritable potentiel vert domestique dont la pérennisation et l'enrichissement pourrait contribuer notablement aux défis environnementaux et alimentaires auxquels font face les populations de la zone étudiée.

La nécessaire amélioration et pérennisation de ce potentiel vert passe par, entre autres, une adhésion complète des populations aux pratiques des UVH. Fort heureusement, d'après les résultats de nos investigations, les représentations associées à 
chacun des UVH sont encourageantes (Figure 10). Néanmoins, nous notons que les populations auraient moins conscience des enjeux paysagers et environnementaux de ces UVH à l'échelle globale comme celle de la commune voire celle de la ville que de ceux de leur micro-espace individuel. La preuve en est le caractère provisoire attribué à la haie vive par $60 \%$ des enquêtés. Il importe donc que soit assuré un travail continu d'éducation environnementale des populations.

\section{Conclusion}

L'analyse effectuée sur les usages du végétal pour l'habitat (UVH) dans la commune périurbaine de Kisenso nous renseigne qu'il y a, dans celle-ci, une diversité d'UVH. Nous observons que la zone géomorphologique de la parcelle, la province d'origine de son propriétaire et le nombre de ménages y vivant sont les facteurs qui présentent le plus d'effet sur la pratique des UVH identifiés, sauf sur celle de l'arbre domestique. La pratique de ce dernier UVH est, en effet, plus généralisée dans la zone.

Par ailleurs, ces divers UVH se composent d'une relative diversité d'espèces végétales et quelques une à deux d'entre elles sont souvent clairement prédominantes. Nous notons, en outre, que les représentations faites de chacun des UVH par les populations sont plus liées à leurs rôles économique et environnemental local qu'aux enjeux paysagers et environnementaux de la grande échelle comme celle de la commune. De plus, il subsiste au sein des populations des représentations préjudiciables à la pratique à long terme de certains UVH dont notamment la haie vive vue comme une clôture provisoire installée par manque de moyens financiers.

Ces résultats illustrent bien les enjeux socio-culturels, économiques et environnementaux de la végétation domestique, c'est-à-dire la végétation présente dans les parcelles habitées, dans la zone périurbaine étudiée. En outre, la présente étude a permis d'enrichir le cadre conceptuel de la végétation domestique à travers le terme $\mathrm{d}$ '« usages du végétal pour l'habitat » permettant de dépasser le terme générique de jardin de case souvent attribué à l'ensemble de la végétation domestique.

\section{CONFLIT D'INTERETS}

Les auteurs déclarent qu'il n'y a aucun conflit d'intérêts.

\section{CONTRIBUTIONS DES AUTEURS}

KRS a proposé le protocole de recherche et réalisé le travail. APM, RO et JB ont supervisé le travail. MST, AKN et ES ont participé à enrichir le manuscrit. Tous les auteurs ont contribué à la rédaction du manuscrit.

\section{REFERENCES}

ActionAid. 2012. La contribution à l'éradication de la pauvreté à Kinshasa. Rapport d'évaluation de depart 2012. ActionAid DRC: République Démocratique du Congo.

Akaike H. 1974. A new look at the statistical model identification. IEEE Transactions on Automatic Control, 19(6): 716 - 723. DOI 10.1109/TAC.1974.1100705.

Bogaert J, Halleux J-M. 2015. Territoires Périurbains: Développement, Enjeux et Perspectives dans les Pays du Sud. Les Presses Agronomiques de Gembloux: Gembloux.

Butler D, Spencer N. 2010. Cities: the century of the city. Nature, 467(7318): 900-901. DOI: 10.1038/467900a.

Cameron RWF, Blanusa T, Taylor J E, Salisbury A, Halstead AJ, Henricot B, Thompson K. 2012. The domestic garden - Its contribution to urban green infrastructure. Urban Forestry and Urban Greening, 11: 129-137. DOI: https://doi.org/10.1016/j.ufug.2012.01.00 2.

CEA, PNUE. 2015. BRICS-Africa partnership for development: driving inclusive growth and transformational change, Addis-Abeba and Nairobi. Commission économique des Nations Unies pour l'Afrique (CEA) et Programme des Nations Unies pour l'environnement (PNUE). http://www.climdev- 
africa.org/document/ Consulté le 17 septembre 2015.

Chalot R. 2015. Écologie et urbanisme : comment les experts du vivant peuventils contribuer à la conception du cadre urbain ? VertigO - la Revue Électronique en Sciences de l'Environnement. DOI: 10.4000/vertigo.16561; http://vertigo.revues.org/16561.

Cook EM, Hall SJ, Larson KL. 2012. Residential landscapes as socialecological systems: a synthesis of multiscalar interactions between people and their home environment. Urban Ecosyst, 15(1): 19 52. https://doi.org/10.1007/s11252-0110197-0.

Dagnelie P. 1998. Statistiques Théoriques et Appliquées (Tomes 1 et 2). De Boeck et Larcier : Brussels.

Dasylva M, Ndour N, Ndiaye O, Sambou B. 2017. Analyse de la flore, de la végétation ligneuse et des fonctions des vallées en zone péri-urbaine post-conflit (Ziguinchor, Sénégal). Int. J. Biol. Chem. Sci., 11(1): 360-377. DOI http://dx.doi.org/10.4314/ijbcs.v11i1.28

FAO. 2010. Développer des Villes plus Vertes en République Démocratique du Congo. Division de la Production Végétale et de la Protection des Plantes. Organisation des Nations Unies pour l'Alimentation et l'Agriculture (FAO) : Rome.

Forthofer RN, Lee ES, Hernandez M. 2007. Biostatistics: a Guide to Design, Analysis and Discovery. Elsevier Acadenic Press: USA.

Hammer S, Kamal-Chaoui L, Robert A, Plouin M. 2011. Cities and Green Growth: A Conceptual Framework. OECD Regional Development Working Papers 2011/08. http://dx.doi.org/10.1787/5kg0tflmzx34en.

Kabir MdE, Webb EL. 2008. Floristics and structure of south-western Bangladesh homegardens. Int. J. Biodiversity Sci. Manag., 4(1): $54 \quad-\quad 64$. https://doi.org/10.1080/17451590809618 183.

Kanda M, Akpavi S, Wala K, Boundjou GD, Akpagana K. 2014.Diversité des espèces cultivées et contraintes à la production en agriculture maraîchère au Togo. Int. J. Biol. Chem. Sci., 8(1): 115-127. DOI : http://dx.doi.org/10.4314/ijbcs.v8i1.11

Kayembe Wa Kayembe M, Wolff E. 2015. Contribution de l'approche géographique à l'étude des facteurs humains de l'érosion ravinante intra-urbaine à Kinshasa (R.D. Congo). Geo-Eco-Trop., 39(1):119-138.

http://www.geoecotrop.be/uploads/publi cations/pub_391_07.pdf

Koubouana F, Ifo S A, Loupet LBM, Ndinga E. 2016. Diversité floristique et dynamique de reconstitution de la forêt du Parc Zoologique sous plantations à eucalyptus à Brazzaville, Congo. Int. J. Biol. Chem. Sci., 10(2): 609-619. DOI : http://dx.doi.org/10.4314/ijbcs.v10i2.13

Latham P, Konda Ku M. 2007. Plantes Utiles du Bas-Congo, République Démocratique $d u$ Congo (2ème édn). Mystole Publication : Royaume-Uni.

Lelo Nzuzi F. 2008. Kinshasa: Ville et Environnement. Edition Harmattan: Paris.

Luginbühl Y. 2012. La Mise en Scène du Monde : Construction du Paysage Européen. Éditions CNRS : Paris.

Makumbelo E, Paulus JJSJ, Luyindula N, Lukoki L. 2005. Apport des arbres fruitiers à la sécurité alimentaire en milieu urbain tropical: cas de la commune de Limete-Kinshasa, République Démocratique du Congo. Tropicultura, 23(4): 245-252. http://www.tropicultura.org/text/v23n4/2 45.pdf

Méndez VE, Lok R, Somarriba E. 2001. Interdisciplinary analysis of homegardens in Nicaragua: microzonation, plant use and socioeconomic importance. Agroforestry Systems, 51(2): 85-96. DOI: 10.1023/A: 1010622430223 
Misilu Mia NE, Chen S, Zhang Li Q. 2010. Sustainable Urbanization's Challenge in Democratic Republic of Congo. Journal of Sustainable Development, 3(2): 242 254.

DOI: http://dx.doi.org/10.5539/jsd.v3n2p 242

Miti TSF, Aloni KJ, Kisangala MM. 2004. Crise morphogénique d'origine anthropique dans le modelé du relief de Kinshasa. Bull. du CRGM, 5(1):1-12.

Ortiz-Sanchez A, Monroy-Ortiz C, RomeroManzanares A, Luna-Cavazos M, Castillo-Espana P. 2015. Multipurpose functions of home gardens for family subsistence. Botanical Sciences, 93(4): 791-806. DOI: 10.17129/botsci.224

Pauwels L. 2003. Les Plantes Vasculaires des Environs de Kinshasa. Editions Pauwels : Bruxelles.

Peel MC, Finlayson BL, Mcmahon TA. 2007. Updated word map of the KöppenGeiger climate classification. Hydrology and Earth System Sciences, 11(5) : 16331644.

Polorigni B, Radji R A, C K. 2015. Politique publique de gestion des espaces verts de la ville de Lomé au Togo. Int. J. Biol. Chem. Sci., 9(4): 1888-1901. DOI : http://dx.doi.org/10.4314/ijbcs.v9i4.14

Rapport de commune. 2015. Rapport de la commune de Kisenso, exercice 2015. Commune de Kisenso : Kinshasa.

Rey F, Ballais J-L, Marre A, Rovéra G. 2004. Rôle de la végétation dans la protection contre l'érosion hydrique de surface. $C$. $R$. Geoscience, 336(11) : 991-998. DOI: 10.1016/j.crte.2004.03.012
Salako KV, Fandohan B, Kassa B, Assogbadjo AE, Idohou AFR, Gbedomon RC, Hakeredza S, Dulloo ME, Glèlè Kakaï R. 2014. Home gardens: an assessment of their biodiversity and potential contribution to conservation of threatened species and crop wild relatives in Benin. Genet Resour Crop Evol, 61: 313-330. DOI: 10.1007/s10722-013-0035-8

Sambiéni KR, Toyi MS, Mama A. 2015. Perception paysanne sur la fragmentation du paysage de la Forêt classée de l'Ouémé Supérieur au nord du Bénin ». VertigO - la revue électronique en sciences de l'environnement, 15(2). http://vertigo.revues.org/16477; DOI : 10.4000/vertigo. 16477

SOSAK. 2014. Schéma d'Orientation Stratégique de l'Agglomération Kinoise (SOSAK) et Plan particulier d'aménagement de la zone Nord de la ville. Groupe Huit/Arter : Kinshasa.

UN Habitat. 2016. Urbanization and development: emerging futures. World cities report: Nairobi.

Wouters T, Wolff E. 2010. Contribution à l'analyse de l'érosion intra-urbaine à Kinshasa (R.D.C). Belgeo, 3 : 293-314. DOI: $10.4000 /$ belgeo.6477.

Yossi H, Kaya B, Traoré CO, Niang A, Butare I, Levasseur V, Sanogo D. 2004. Les Haies Vives au Sahel: État des Connaissances et Recommandations pour la Recherche et le Développement. World Agroforestry Centre : Nairobi. 